\title{
Transforming Practice with HOPE (Healthy Outcomes from Positive Experiences)
}

\author{
Dina Burstein ${ }^{1} \cdot$ Chloe Yang $^{1} \cdot$ Kay Johnson ${ }^{2} \cdot$ Jeff Linkenbach $^{3} \cdot$ Robert Sege $^{1}$ \\ Accepted: 26 April 2021 / Published online: 5 May 2021 \\ (c) The Author(s), under exclusive licence to Springer Science+Business Media, LLC, part of Springer Nature 2021
}

Keywords Child abuse · Covid-19 · Positive childhood experiences · Adverse childhood experiences

\section{Purpose}

Positive childhood experiences (PCEs) have profound effects on health and development, buffering against and preventing the harms of toxic stress (Bethell et al., 2019). Efforts to translate this science into practice are needed and will improve the effectiveness of current approaches that focus on identifying risks and specific child and family problems.

Healthy outcomes from positive experiences (HOPE) translates research findings about the effects of PCEs into clinical and organizational changes that support optimal development and resilience in the face of adversity. This paper describes the growing evidence that supports the health effects of positive childhood experiences, and uses these insights to promote a paradigm shift that will incorporate the identification, celebration, and support of PCEs as an integral part of caring for children and families. The HOPE-informed approach expands current care, shifting

Dina Burstein

dburstein@tuftsmedicalcenter.org

Chloe Yang

cyang3@tuftsmedicalcenter.org

Kay Johnson

kay.johnson@johnsongci.com

Jeff Linkenbach

jeff@montanainstitute.com

Robert Sege

rsege@tuftsmedicalcenter.org

1 Center for Community-Engaged Medicine, Tufts Medical Center, Institute for Clinical Research and Health Policy Studies, 800 Washington Street, Boston, MA 02111, USA

2 Johnson Group Consulting, Inc., Hinesburg, USA

3 The Montana Institute, PO Box 5015, Bozeman, MT 59717, USA from a sole focus on adverse childhood experiences (ACEs) toward an additional focus on PCEs (Yang, 2021).

Our understanding of the effects of experience on child development has advanced since the seminal 1998 ACEs study, which linked child maltreatment with poor adult health (Felitti et al., 1998; Hughes et al., 2017). Many of these ACEs themselves result from adverse community experiences, which notably include systemic racism, historic trauma, and ongoing inequities in social determinants of health (Ellis \& Dietz, 2017). HOPE adds a balancing lens to this focus on ACEs-one that includes consideration of both adverse community experiences, including effects of systemic racism, and the individual and organizational structures that have arisen to address their effects.

Recent research has shown that anatomic and physiologic changes in the developing brain also occur in response to positive stimuli and events (Kwak et al., 2019; Tomlinson et al., 2016), and that the brain shows growth and healing following a significant traumatic event (Fujisawa et al., 2015; Nakagawa et al., 2016). These and other observations led to the study of PCEs alongside ACEs, shifting the focus to a relationship-based, Science of the Positive (Linkenbach, 2020), approach which touches on all levels of the socialecological system (Bronfenbrenner, 2009).

The COVID-19 pandemic raised concerns that many children might be at increased risk of maltreatment due to increased family stress from economic hardship, fears of contracting the virus, and social isolation (Silva, 2020). Children have been separated from friends and group education settings, their routines have been disrupted, and many no longer have access to grandparents as caregivers. There have been reports of increased child behavior and mental health problems (Patrick et al., 2020), as well as concerns raised about the increased risk of child maltreatment, despite the observed dramatic decrease in child maltreatment reports (U.S. Department of Health \& Human Services, 2020). 
However, viewed from the lens of HOPE and considering PCEs, many families have grown closer as they have spent more time together, particularly among those whose economic stress has been addressed with unemployment benefits.

\section{Description}

The HOPE framework focuses on key PCEs. This paper describes the development of that framework and how it has adapted to respond to needs presented by the COVID19 pandemic. The framework complements other researchbased approaches, including the centers for disease control and prevention's (CDC's) Essentials for Childhood initiative, which focuses on policy and cultural norms (Centers for Disease Control \& Prevention, 2019), and the Strengthening Families Approach (Center for the Study of Social Policy, 2020), ${ }^{\mathrm{TM}}$ which focuses on families.

\section{Development of the HOPE Framework}

Translational research seeks to transform research findings into practices to improve individual and public health. HOPE was developed as a confluence of systematic research and stakeholder input (Sege et al., 2017a). Research results have been previously reported (Bethell et al., 2019; Sege, 2017b). Development of the HOPE framework just prior to the pandemic relied on three steps: (1) distilling research results into messages and infographics to seed stakeholder conversations, (2) systematically engaging key stakeholders in strategic planning, and (3) deploying a dissemination strategy for multiple audiences.

\section{Distilling Research Results to Seed Stakeholder Conversations}

We studied PCEs using a 7-item scale developed and implemented with the Wisconsin Behavioral Risk Factor Surveillance Survey. The scale was adapted from the previously validated child and youth resilience measure (CYRM-28) (Ungar, 2011). (See Table 1). As reported in our 2019 article in JAMA pediatrics, these items had the psychometric properties allowing us to develop a PCE score. More generally, similar results have been obtained with adolescents (counter-ACEs) and with a clinical scale for use in early childhood (benevolent childhood experiences). Analysis of these survey results demonstrated a dose-response association between the number of PCEs reported and the extent to which these protective factors reduced the likelihood of adult depression or poor mental health.
Table 1 Items added to the Wisconsin Behavioral Risk Factor Surveillance Survey, based on the previously validated child and youth resilience measure (CYRM-28)

\begin{tabular}{ll}
\hline How often or how much as a child did you \\
\hline 1 & Feel able to talk to family about feelings \\
2 & Feel your family stood by you during difficult times \\
3 & Enjoy participating in community traditions \\
4 & Feel a sense of belonging in high school \\
5 & Feel supported by friends \\
6 & Have at least two nonparent adults who took genu- \\
7 & ine interest in you \\
\hline
\end{tabular}

a Answer choices for these items were: never, rarely, sometimes, often, or very often

${ }^{b}$ Not including those who did not attend school or were home schooled

Concepts central to HOPE were developed via a 2017 literature review, which examined multiple evidence-based interventions and found common elements in their core concepts. The infographic shown as Fig. 1 illustrates the four evidence-based themes, which are the building blocks-or key PCEs and their sources-central to the HOPE framework: (1) relationships with adults and other children, (2) safe, stable, and equitable environments to live, learn and play, (3) social/civic engagement, and (4) social/emotional growth (Sege, 2017b).

\section{Engaging Key Stakeholders}

Stakeholder input was garnered through (1) workshops at locations throughout the United States, and (2) the HOPE national advisory board (NAB), formed to help develop the HOPE strategic plan for implementation and dissemination.

In order to root the HOPE framework in actual organizational and professional needs, we conducted workshops and presentations with practitioners, organizational leaders, and government officials throughout the country. We held in-depth workshops with three groups of key stakeholders: (1) three sessions hosted by the California ACEs Connection, (2) an all-day meeting at the National Center for Injury Prevention and Control at the CDC in Atlanta, and (3) meetings with leadership from Prevent Child Abuse America and Healthy Families America. Our goal was to solicit feedback on how workshop participants perceived the value of HOPE as a shift in paradigm, how HOPE might fit into their work, and what specific tools and approaches they would need to adopt the HOPE framework. We queried participants with direct questions and collected their input systematically. A major purpose of our presentations and workshops was to garner input on how to move the HOPE program forward 


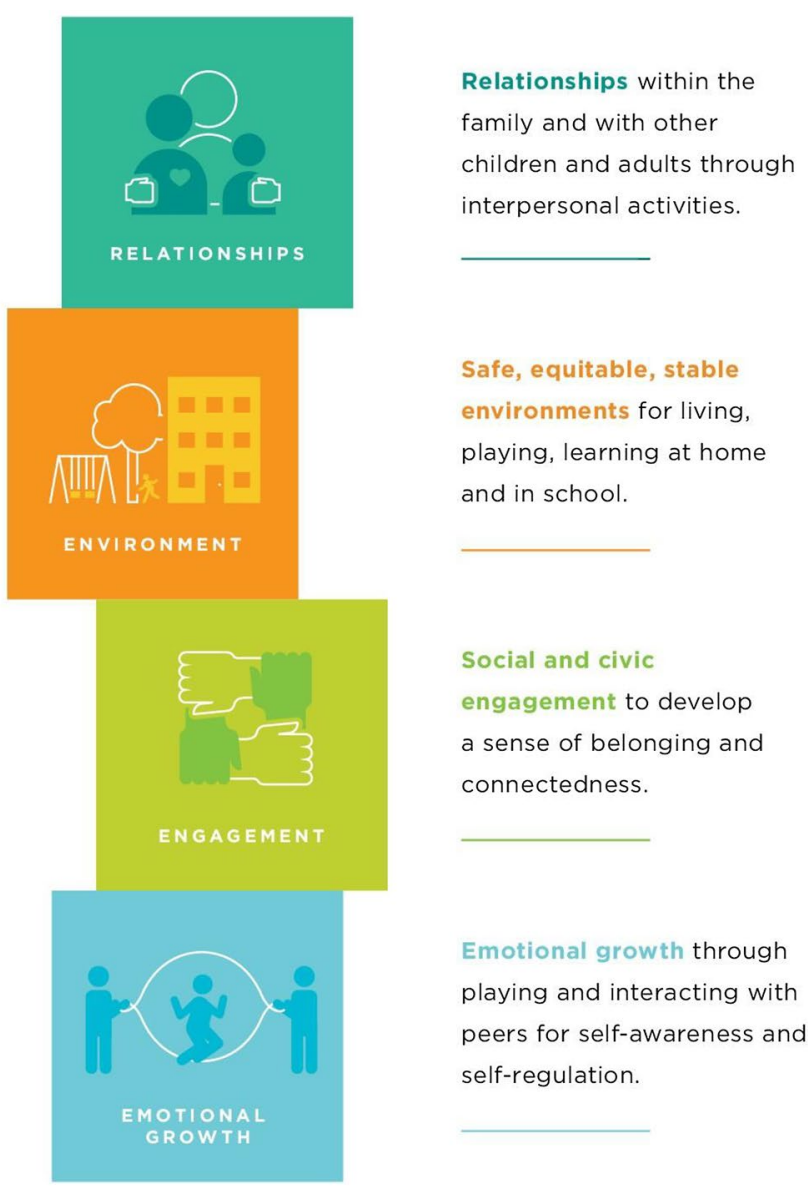

Fig. 1 HOPE Building Blocks: This infographic summarizes key findings from a 2017 literature review by Sege and Harper Browne, which identified common elements in multiple evidence-based interventions. These themes are central to the HOPE framework and make up the four building blocks of HOPE, or key positive childhood experiences and their sources: (1) relationships, (2) safe, stable, and equitable environments, (3) social/civic engagement, and (4) social/emotional growth might incorporate the framework of HOPE into their work.

To invite guidance from leaders in the fields of clinical medicine, child abuse prevention, public health, and health equity, HOPE personnel convened a NAB. The board met three times during the first half of 2020, to advise and guide the HOPE project team on strategic planning, dissemination, and development activities. This group also facilitated connections between the HOPE team and additional relevant organizations and stakeholders, accelerating initial dissemination of the HOPE framework.

\section{Deploying a Dissemination Strategy}

HOPE relies on three major dissemination avenues: (1) in person or virtual trainings and workshops, (2) digital engagement, and (3) earned media. HOPE launched a website, https://positiveexperience.org, in February 2020, an ACEs connection community page in March 2020, and a newsletter in June 2020 (which one can subscribe to on the HOPE website). Finally, HOPE has developed an earned media presence through national outlets, including PBS NewsHour, CNN.com, and the Wall Street Journal. The website has received over 25,000 visitors from across the country and serves as a hub for HOPE materials, updates, and resources. As of April 2021, 1072 individuals are subscribed to the newsletter, and the ACEs Connection community contains 118 members. A complete list of HOPE dissemination strategies and reach are listed in Table 2.

\section{Refining the HOPE Framework and Adaptation for COVID-19}

Engagement with key stakeholders led to substantial refinement of the HOPE model and strategic plan. The following principles arose from stakeholder engagement:

from theory into practice, and specifically, how attendees

Table 2 HOPE dissemination strategies, activities, and corresponding reach from March 2020-October 2020

\begin{tabular}{llc}
\hline Dissemination strategy & Activities & Reach \\
\hline Workshops & 20 virtual workshops/trainings & $\sim 2,500$ participants \\
Website (positiveexperience.org) & 3 new pages, 1 new worksheet, 2 worksheets translated into 6 additional & 26,070 views \\
& languages, 5 new HOPE resources & 12,665 views ACEs \\
Blog* & 43 new blog posts, including 23 Covid-19 related blog posts & 96 community members \\
ACEs connection community & 40 new community posts & 392 subscribers, 1630 \\
HOPE newsletter** & 5 newsletters sent & total times viewed \\
Earned media & PBS NewsHour, CNN.com, USA today, and more & 7 media appearances \\
\hline
\end{tabular}

*All blogs can be viewed at positiveexperience.org

**Subscribe to the HOPE newsletter here (https://mailchi.mp/d000afc02212/hope-mailing-list) 
- The HOPE framework and language can serve as a bridge between health, family support, and social services sectors.

- HOPE can be embedded into existing community outreach and community action efforts, including those focused on ACEs and trauma-informed care.

- Providers want community and individual skill-building tools to assist them with incorporating HOPE into their work.

Frontline providers, including pediatricians, home visitors, and substance use prevention counselors, needed to pivot from in person or in school direct interactions to virtual sessions with clients. Providers themselves also experienced disruptions in their childcare and expressed increased concerns about their older relatives. Many became quite concerned about their clients. This created a need and desire for assistance in successfully making this pivot to virtual platforms. The HOPE team responded to numerous requests for training and technical assistance and has conducted a total of 21 COVID-19 focused webinars and virtual workshops, reaching a total of approximately 2,500 individuals. These highly interactive events gave participants the opportunity to share how they are already using HOPE in their work, as well as barriers and facilitators to implementing HOPE.

In order to focus attention on the effectiveness of PCEs in supporting child development during the pandemic, we published 23 COVID-19 related blog posts to the HOPE website, ACEs Connection main page, and the balancing ACEs with HOPE community on ACEs connection. Some of these blog posts were also cross-posted to the websites of the Center for the Study of Social Policy and the Early Childhood Connector, hosted by Start Early.

Using the HOPE approach, the initial focus of these blog posts was to provide tips for how to manage in these difficult times, emphasizing opportunities for positive experiences presented by the COVID-19 crisis, specifically those related to families, children, and adolescents. Some blog posts were written as advice directly to families, and others were written for providers who serve families. Our most read post to date is "Love in the time of Coronavirus: a HOPEinformed guide for parents". This post has been viewed more than 2,400 times. Dr. David Willis (pediatrician and Senior Fellow at the Center for the Study of Social Policy) wrote a blog post about how foundational relationships early in life provide support for lifelong health and development, with emphasis on how to encourage strong foundational relationships, even in a period of physical distancing and social isolation. Pediatricians Dr. Heather Forkey and Dr. Moira Szilagyi, president-elect of the American Academy of Pediatrics, were interviewed about the importance of understanding and promoting resilience and positive experiences during the pandemic. Policy leaders Dr. Charles
Bruner and Kay Johnson provided insights regarding how public programs such as Medicaid and the Title V Maternal and Child Health Services Block Grant can support action to advance the HOPE approach. Nine additional blogs specifically addressing COVID-19 issues have attracted nearly 3,000 views collectively. All blog posts are available on the HOPE website (Yang, 2021).

As the movement for racial justice grew, we added content related to specific challenges and inequities exacerbated by COVID-19, often written from interviews with experts who provided key insights. The following examples give a sense of the tone and focus of these blog pieces: Corey Best (a member of the HOPE NAB and leader of the Birth Parent National Network, Children's Trust Fund Alliance) spoke on fatherhood, as well as the disparities and racism illuminated by the pandemic. Dr. Danielle Laraque-Arena (a member of the HOPE NAB, a senior scholar-in-residence at the New York Academy of Medicine, professor and president emerita at SUNY Upstate Medical University, pediatrician, and adjunct professor of epidemiology at Columbia University) spoke about inequities that have been exacerbated by COVID-19, including access to health care, poverty, and mental health needs. The Reverend Darrell Armstrong (pastor of the Shiloh Baptist Church of Trenton, NJ) shared his views as a national leader on implementing the Strengthening Families approach, as well as communities' lack of access to food, technology, and mental health services during the COVID-19 crisis.

Additionally, amidst growing national concerns about family life during the pandemic, HOPE garnered a great deal of earned media attention related to COVID-19. Earned media, in the context of this article, refers to public media coverage of an issue, program, or campaign that is of public interest and is broadcast by different media outlets. This is in contrast to paid media or conventional advertising (Kornfield et al., 2015). For HOPE, earned media included features in the Wall Street Journal, CNN.com, and PBS NewsHour. These opportunities helped spread the general message of family and community support for child development, resulting in increased awareness of the HOPE strengths-based approach.

\section{Assessment}

Recent research has pointed to PCEs as protective and mitigating factors against the effects of ACEs and toxic stress (Bethell et al., 2019). Neurobiological studies, showing physical and functional changes in the brain in response to positive stimuli, suggest a physiologic mechanism for this phenomenon (Fujisawa et al., 2015; Kwak et al., 2019; Lake et al., 2017; Nakagawa et al., 2016; Nenert et al., 2018; Tomlinson et al., 2016). Mitigating toxic stress is especially 
urgent during the pandemic, as families and children experience social isolation, financial difficulty, and other disruptions to their daily lives, factors which also increase risk of child maltreatment. In this paper, we present a description of both the translation of these research findings into a robust, multifaceted, and strengths-based program, as well as the adaptation of this program to respond to the COVID19 pandemic.

Prior research in child abuse prevention has demonstrated the importance of family strengths, attention to early foundational relationships (Bethell et al., 2016; Browne, 2014; Harper Browne, 2016), and the close association of child abuse with other aspects of violence in the home (Flaherty et al., 2010). Promoting PCEs can contribute to reductions in the incidence of child abuse and neglect by offering families practical information about family and child needs (Harper Browne, 2016). Our work promoted PCEs and knowledge of their value during COVID-19, using new outreach strategies specifically adapted to messaging during the pandemic.

Content was developed through consistent focus on the four building blocks of HOPE. This framework and the building blocks directed guidance for practitioners working with families, as well as for parents and caregivers directly. Emphasis was placed on parents' and providers' ability to both create positive experiences for children and reduce the anxiety and stress associated with the COVID-19 crisis and consequent stressors. This work particularly highlighted challenges faced by marginalized families and opportunities to advance equity, through trainings and dissemination platforms. Growing interest in using strengths-based approaches and in applying an equity lens to working with families created a readiness and desire for more information about HOPE, allowing for quick mobilization to help address issues presented by the COVID-19 crisis. Additional research is needed to better understand how families have adapted to the pandemic and the resulting social isolation and economic challenges.

\section{Conclusion}

The HOPE framework transforms interactions between providers and parents in multiple settings-including health care, early childhood education, home visiting, child welfare, and other public health efforts. By opening up dialogue to focus on a family's assets and strengths, as well as respecting the cultural and individual expertise of parents and families, HOPE can better align family and provider goals and priorities. The HOPE framework provides a balanced approach to communication, assessment, workflow, and true partnerships between providers and families that may promote PCEs, help prevent child abuse and neglect, and break the link between adversity and toxic stress. We see the potential for state agencies concerned with child health and well-being, including Title V Maternal and Child Health Programs, to support and advance this work through training for primary health care providers, home visitors, community health workers, early educators, and others. The long-term goal of HOPE is a paradigm shift in trauma-informed care toward HOPE-informed prevention, as we seek to advance equity in services for children and their families, to support strengths in addition to identifying risks, to equalize power between providers and families in the decision-making process, and ultimately, to improve lifelong mental and physical health outcomes.

Acknowledgements The authors acknowledge the JPB Foundation for their generous support of the HOPE project. The project described was supported by the National Center for Advancing Translational Sciences, National Institutes of Health, Award Number UL1TR002544. The content is solely the responsibility of the authors and does not necessarily represent the official views of the NIH.

\section{Declarations}

Conflicts of interest The authors declare they have no conflict of interest.

\section{References}

Bethell, C., Gombojav, N., Solloway, M., \& Wissow, L. (2016). Adverse childhood experiences, resilience and mindfulness-based approaches: Common denominator issues for children with emotional, mental, or behavioral problems. Child and Adolescent Psychiatric Clinics of North America, 25(2), 139-156. https://doi.org/ 10.1016/j.chc.2015.12.001

Bethell, C., Jones, J., Gombojav, N., Linkenbach, J., \& Sege, R. (2019), Positive childhood experiences and adult mental and relational health in a statewide sample: associations across adverse childhood experiences levels. JAMA Pediatrics, 173(11), e193007. https://doi.org/10.1001/jamapediatrics.2019.3007

Bronfenbrenner, U. (2009). The ecology of human development: experiments by nature and design. Havard University press.

Browne, C. (2014). The strengthening families approach and protective factors framework: Branching out and reaching deeper. Center for the Study of Social Policy. Retrieved October 27, 2020, from https://cssp.org/wp-content/uploads/2018/11/Branching-Out-andReaching-Deeper.pdf

Center for the Study of Social Policy. (2020). Strengthening families: increasing positive outcomes for children and families. Retrieved August 11, 2020, from https://cssp.org/our-work/project/stren gthening-families/

Centers for Disease Control and Prevention. (2019). Essentials for childhood: Creating safe, stable, nurturing relationships and environments. Retrieved August 19, 2020, from https://www.cdc. gov/violenceprevention/childabuseandneglect/essentials.html

Ellis, W. R., \& Dietz, W. H. (2017). A new framework for addressing adverse childhood and community experiences: The building community resilience model. Academic Pediatrics, 17(7S), S86S93. https://doi.org/10.1016/j.acap.2016.12.011

Felitti, V. J., Anda, R. F., Nordenberg, D., Williamson, D. F., Spitz, A. M., Edwards, V., Koss, M. P., \& Marks, J. S. (1998). Relationship 
of childhood abuse and household dysfunction to many of the leading causes of death in adults. The adverse childhood experiences (ACE) study. American Journal of Preventive Medicine, 14(4), 245-258. https://doi.org/10.1016/s0749-3797(98)00017-8

Flaherty, E. G., Stirling, J., Jr., American Academy of Pediatrics, \& Committee on Child Abuse \& Neglect. (2010). Clinical report-the pediatrician's role in child maltreatment prevention. Pediatrics, 126(4), 833-841. https://doi.org/10.1542/peds.2010-2087

Fujisawa, T. X., Jung, M., Kojima, M., Saito, D. N., Kosaka, H., \& Tomoda, A. (2015). Neural basis of psychological growth following adverse experiences: A resting-state functional MRI study. PLoS ONE, 10(8), e0136427. https://doi.org/10.1371/journal. pone. 0136427

Harper Browne, C. (2016). The strengthening families approach and protective factors framework ${ }^{\mathrm{TM}}$ : A pathway to healthy development and well-being. In C. J. Shapiro \& C. Harper Browne (Eds.), Innovative approaches to supporting families of young children. (pp. 1-24). Springer Internationl publishing.

Hughes, K., Bellis, M. A., Hardcastle, K. A., Sethi, D., Butchart, A., Mikton, C., Jones, L., \& Dunne, M. P. (2017). The effect of multiple adverse childhood experiences on health: A systematic review and meta-analysis. Lancet Public Health, 2(8), e356-e366. https:// doi.org/10.1016/S2468-2667(17)30118-4

Kornfield, R., Smith, K. C., Szczypka, G., Vera, L., \& Emery, S. (2015). Earned media and public engagement with CDC's “Tips from Former Smokers" campaign: An analysis of online news and blog coverage. Journal of Medical Internet Research, 17(1), e12. https://doi.org/10.2196/jmir.3645

Kwak, S., Lee, T. Y., Jung, W. H., Hur, J. W., Bae, D., Hwang, W. J., Cho, K. I. K., Lim, K. O., Kim, S. Y., Park, H. Y., \& Kwon, J. S. (2019). The immediate and sustained positive effects of meditation on resilience are mediated by changes in the resting brain. Frontiers in Human Neuroscience, 13, 101. https://doi.org/10. 3389/fnhum.2019.00101

Lake, E. M. R., Bazzigaluppi, P., Mester, J., Thomason, L. A. M., Janik, R., Brown, M., McLaurin, J., Carlen, P. L., Corbett, D., Stanisz, G. J., \& Stefanovic, B. (2017). Neurovascular unit remodelling in the subacute stage of stroke recovery. NeuroImage, 146, 869-882. https://doi.org/10.1016/j.neuroimage.2016.09.016

Linkenbach, J. (2020). What is the science of the positive? . Retrieved 13, July 2020, from http://www.montanainstitute.com/what-is-thescience-of-the-positive/

Nakagawa, S., Sugiura, M., Sekiguchi, A., Kotozaki, Y., Miyauchi, C. M., Hanawa, S., Araki, T., Takeuchi, H., Sakuma, A., Taki, Y., \&
Kawashima, R. (2016). Effects of post-traumatic growth on the dorsolateral prefrontal cortex after a disaster. Science and Reports, 6, 34364. https://doi.org/10.1038/srep34364

Nenert, R., Allendorfer, J. B., Martin, A. M., Banks, C., Vannest, J., Holland, S. K., Hart, K. W., Lindsell, C. J., \& Szaflarski, J. P. (2018). Longitudinal fMRI study of language recovery after a left hemispheric ischemic stroke. Restorative Neurology and Neuroscience, 36(3), 359-385. https://doi.org/10.3233/RNN-170767

Patrick, S. W., Henkhaus, L. E., Zickafoose, J. S., Lovell, K., Halvorson, A., Loch, S., Letterie, M., \& Davis, M. M. (2020). Wellbeing of parents and children during the COVID-19 pandemic: A national survey. Pediatrics. https://doi.org/10.1542/peds. 2020-016824

Sege, R., Bethell, C., Linkenbach, J., Jones, J., Klika, B., \& Pecora, P. J. (2017a). Balancing adverse childhood experiences (ACEs) with HOPE: New insights into the role of positive experience on child and family development. Boston, MA: The Medical Foundation

Sege, R., \& Harper Brown, C. (2017b). Responding to ACEs with HOPE: Health outcomes from positive experiences. Academic Pediatrics, 17, S79-S85

Silva, C. D. (2020). Texas hospital child abuse cases rise in COVID19 outbreak: 'It's hard to think that it's just coincidental'. https:// www.newsweek.com/texas-hospital-child-abuse-cases-rise-covid19-outbreak-1493642. Accessed 10 January 2021

Tomlinson, L., Leiton, C. V., \& Colognato, H. (2016). Behavioral experiences as drivers of oligodendrocyte lineage dynamics and myelin plasticity. Neuropharmacology, 110(Pt B), 548-562. https://doi. org/10.1016/j.neuropharm.2015.09.016

U.S. Department of Health \& Human Services. (2020). National data archive on child abuse and neglect. Retrieved July 13, 2020, from https://www.ndacan.acf.hhs.gov/index.cfm

Ungar, M., \& Liebenberg, L. (2011). Assessing resilience across cultures using mixed methods: construction of the child and youth resilience measure. Journal of Mixed Methods Research, 5(2), $126-149$

Yang, C. (2021). Spreading HOPE Publication Launch.. https://posit iveexperience.org/spreading-hope-publication-launch/. Accessed 12 April 2021

Publisher's Note Springer Nature remains neutral with regard to jurisdictional claims in published maps and institutional affiliations. 